\title{
Aptamer: A Novel Therapeutic Oligonucleotide
}

\begin{abstract}
The aptamer is an oligonucleotide which is a short version of biological nucleic acids (such as DNA and RNA) with defined sequence of nucleotides. Based on the complexity of molecule, the aptamers lies in-between protein molecules and small chemical molecules. Aptamers have high specificity and affinity towards target proteins. They are screened from random sequences of oligonucleotides based on the highest affinity for target proteins using the SELEX (Systematic Evolution of Ligands by Exponential Enrichment). Researchers have discovered various applications of aptamers that are ready to replace the therapeutic use of biological proteins (such as antibodies) that have complexity in manufacturing and characterization. The present review describes the structural modification in aptamers such as PEGylation, substitution of functional groups, use of an enantiomeric oligonucleotide and its applications. Aptamers can be deactivated, when needed, by the use of reversal agent that contains oligonucleotide sequence complementary to aptamers. This property of aptamers makes them potential therapeutic from a safety point of view. In recent scenario, aptamers are developed for targeted drug delivery systems by conjugating with drug molecules or delivery vesicles such as liposomes. 'Anti-sense Aptamers' are being developed to silent the expression of genes responsible for the proliferative growth of tissue in cancer. Other applications of aptamers such as environmental monitoring and laboratory testing are also described in this review.
\end{abstract}

Keywords: Aptamer; Oligonucleotide; Biosensor; Anti-sense aptamer; Aptasome; Biotechnology; Liposome; Anti-cancer
Review Article

Volume 5 Issue 6 - 2017

Nrupa Patel ${ }^{1 *}$ and Sandipkumar Patel ${ }^{2}$

${ }^{1}$ Product Development, Teligent Pharma Inc., USA

${ }^{2}$ Formulation Development, Navinta LLC, USA

*Corresponding author: Nrupa Patel, Product Development, Teligent Pharma Inc., NJ. USA 08310, Email: nrupapharma2016@gmail.com

Received: June 10, 2017 | Published: July 11, 2017
Abbreviations: CASTing: Cyclic Amplification and Selection of Target; DNA: Deoxyribonucleic Acid; FCS: Fluorescence Correlation Spectroscopy; fM: Femtomolar; LFA: Lateral Flow Assay; ODN: Oligodeoxynucleotide; PCR: Polymerase Chain Reaction; PSMA: Prostate-Specific Membrane Antigen; RNA: Ribonucleic Acid; SAAB: Selected and Amplified Binding site; SELEX: Systematic Evolution of Ligands by Exponential Enrichment; SWNT: SingleWalled Carbon Nanotubes; VPF: Vascular Permeability Factor

\section{Introduction}

Biotechnology based products are incredibly capturing market shares day by day. Chemistry based synthesis has a limitation when we talk about the tertiary and quaternary structure of large molecules such as protein. The human body is functioning by different biochemical reactions. Protein molecules are broadly involved in every aspect of the biological reaction. In the current paradigm, such functional proteins get expressed in microorganism by genetic variation as a part of the upstream process. Subsequently, these proteins are separated from the microorganisms and purified (as a downstream process) retaining its biological activity to inject in the human body. These complex proteins are difficult to cultivate, separate, characterize and reproduce $[1,2]$.

The basic mechanism of protein synthesis in any of the living organism involves transcription and translation of polynucleotide chain of Adenine, Guanine, Thymine, and Cytosine [3]. The sequence of the oligonucleotide is responsible for translating specific protein using a specific sequence of amino acid involved in the synthesis of protein. Currently marketed protein formulations readily provide biological action at the target site when injected into the body. Such protein molecules are produced outside of the human body and injected in purified and active form. However, the stability of proteins is the major concern in the development of protein formulations. Such biological molecules have a tertiary and quarternary structure with different sensitive bonding such as disulfide bond, hydrogen bond, and amide bond. Antibodies are widely used as protein formulations approved by USFDA and are available in the market [4]. Due to difficulties in development, scale-up, reproducibility, and characterization issues, there should be an alternative to protein formulations.

Aptamers are good candidates that can serve both as simple chemical molecules with regards to synthesis and characterization, and protein molecules for functional properties in the biological system [5]. Aptamers are oligonucleotide chain (of DNA or RNA) consisting individual nucleotides (Adenine, Guanine, Thymine, and Cytosine) linked with phosphodiester bond presented in Figure 1 . They have very high affinity for particular protein. Let's understand how they are useful therapeutically. As mentioned, Aptamers have very high affinity for the specific target protein; they can bind to protein available on the cell surface, blood stream or in any tissue where they can reach upon administration [6]. Schematic representation of Aptamer binding with target protein is presented in Figure 2. It can alter the activity of the specific protein, or it can bind to any ligand or receptor and provide antagonistic effect to receptor-mediated pharmacological effect [2]. Some of the therapeutic applications of Aptamers include targeting to various diverse physiological conditions to receptor proteins or mediators. 


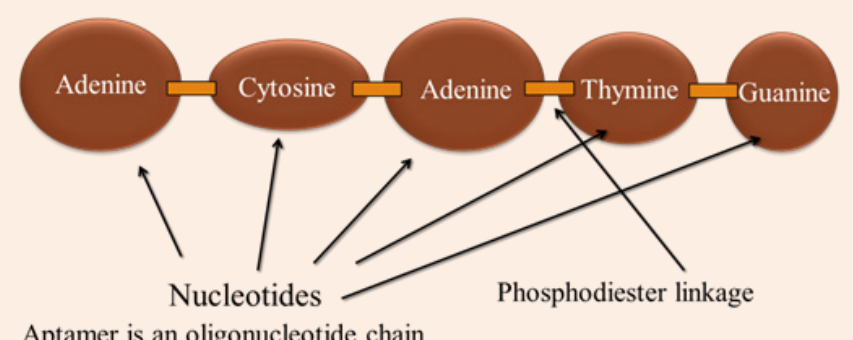

Aptamer is an oligonucleotide chain

$($ Oligo $=$ few , nucleotide $=$ Adenine, Guanine, Thymine, Cytosine $)$

Figure1: Basic structure of Aptamer.

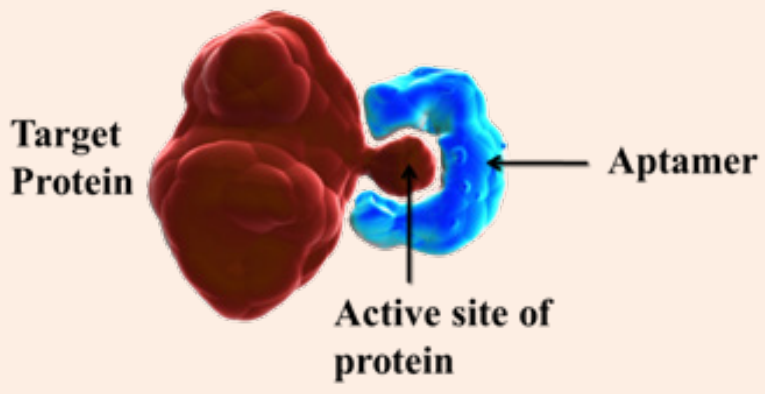

Figure2: Schematic representation of Aptamer binding with target protein.

\section{How are aptamers produced?}

An important aspect of Aptamers is the commercial feasibility of manufacturing. Production of protein is quite complex and requires micro-organisms as a host cell that increases the chance of biological contamination of viruses and hosts cell DNA. Aptamers are oligonucleotides which are chemically synthesized with a specific sequence of nucleotides. Systematic Evolution of Ligand by Exponential Enrichment (SELEX) is the reported applied technology for Aptamers synthesis [7]. SELEX is a combinatorial chemistry for producing oligonucleotides of specific sequence based on homing principle. Researchers have also referred as Selected and Amplified Binding (SAAB) site or cyclic amplification and selection of target (CASTing) [8,9]. In the initial stage, very large number of nucleotide library is synthesized with fixed number of nucleotide chain (n). For each position of a nucleotide, there are four possibilities which can generate $4^{\mathrm{n}}$ sequences of the oligonucleotides. These oligonucleotides act as Primer. Prepared oligonucleotide library is then exposed to the immobilized target protein, where the ones with a high affinity for target protein gets retained, and rest of the sequences gets eluted. Retained sequence is further separated and amplified using Polymerase Chain Reaction (PCR) [10]. The resulted aptamers have very high affinity for a particular protein which can act as therapeutics (Figure 3).

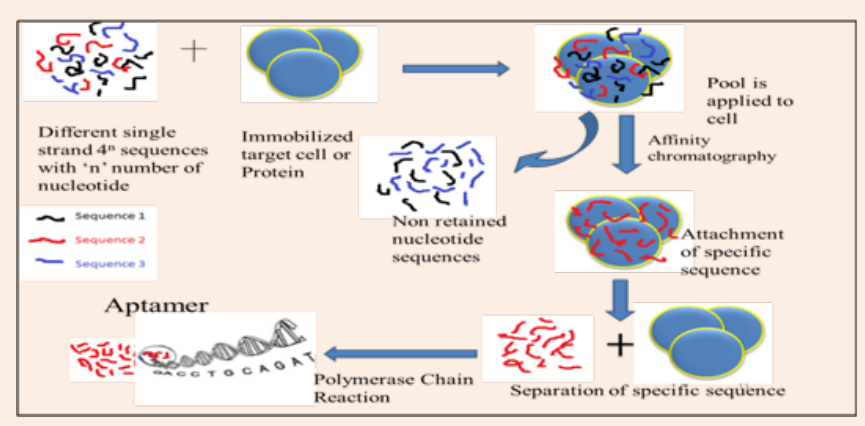

Figure3: Schematic representation of SELEX for Aptamer production.

\section{Inactivation of aptamers}

Once we administer therapeutic protein, it is very difficult to stop its action on the target site. However, the complementary sequence of the aptamer is possible to construct, which can act as reversal agent and irreversibly bind with specific aptamers and denature them by neutralization. In Figure 4, reversal agent with a complementary sequence to aptamer binds to an aptamer which is attached to the target protein. Thus, the therapeutic effect of the aptamer is reversible in nature where the reversal agent can inhibit the effect of aptamers $[11,12]$.

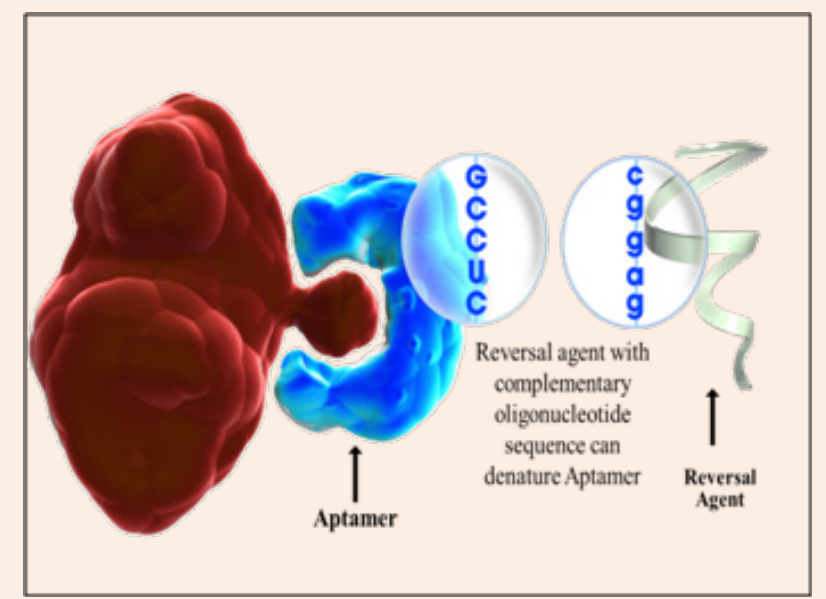

Figure4: Inactivation of Aptamer by reversal agent.

\section{Modified aptamers}

Structurally modified aptamers are constructed by incorporating modified nucleotide into SELEX process. Such modification includes incorporation of individual modified nucleotide (e.g. 2'-amino pyrimidines, 2'-fluoropyrimidines, 2 '-0-methyl ribose purines and pyrimidines, etc.) or completely substituting nucleotide $[13,14]$. The linkage between nucleotide can also be modified (e.g. phosphorothioate linkage). Compounds 
with higher molecular mass can be incorporated (such as Polyethylene glycol) by conjugating with 5'-terminal. Aptamer with high molecular weight reduces the elimination and hence increases the half-life. In one reported study, bioavailability of Aptamers was increased by conjugation with cholesterol or polyethylene glycol. Such conjugation resulted into increased halflife to several hours (using cholesterol) to days and months (using PEG) [15]. L-nucleotides can be used instead of naturally occurring
D-nucleotide. Such nucleotides are also known as Spiegelmers, a German word for mirror. The modification in chirality of the nucleotides from Dextro to the Levo form in the Spiegelmers eliminates the ability of the nuclease enzymes to recognize them and make them nuclease resistant [16]. Thus, modification in aptamers improves stability, better protection against nuclease enzyme and higher affinity for target protein (Figure 5).

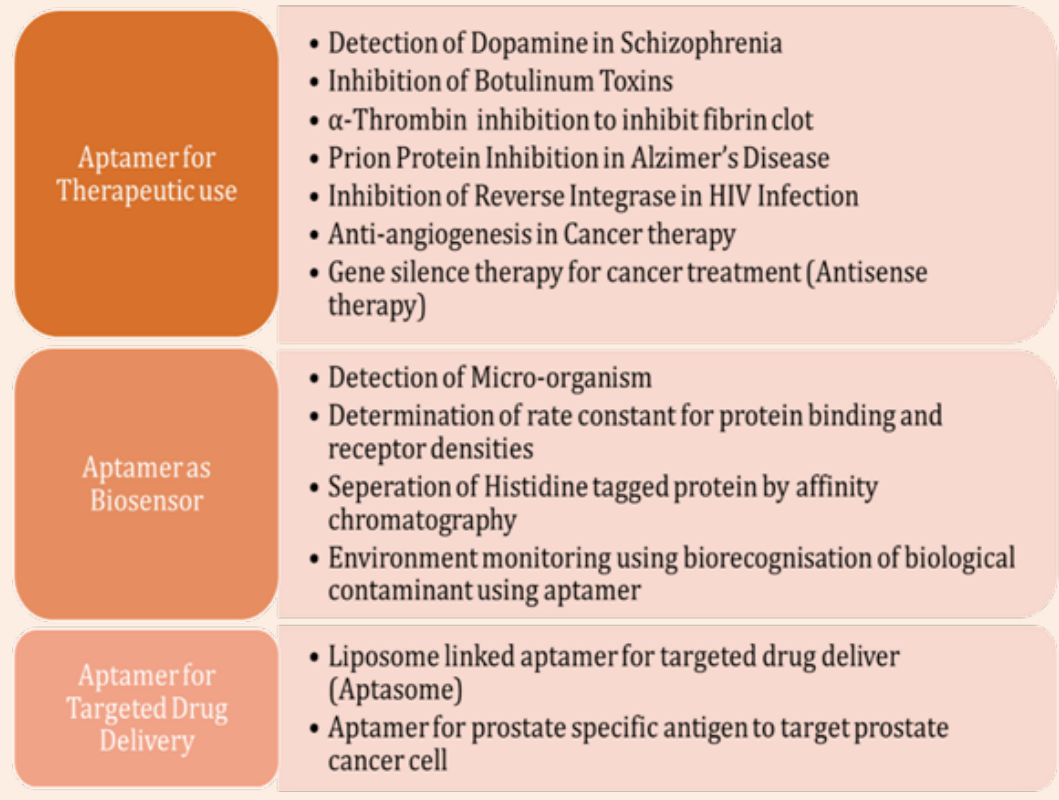

Figure5: Schematic presentation describing some applications of aptamer.

\section{Therapeutic Applications}

All kind of receptors are made up of proteins and have an affinity for the specific mediator, ligand or physio-chemical changes. The pharmacological action can be modified by inhibiting certain targets such as receptor or mediator. Systemically designed Aptamer can specifically bind to target protein and alter its action. Aptamers are reported for detection of dopamine and treatment of schizophrenia $[17,20]$. Botulinum toxin is one of the most devastating toxin reported. DNA and RNA aptamers have been designed to inhibit the toxicity of Botulinum toxin [18-20].

In various disease conditions, proteins play an important role as a mediator. $\alpha$-Thrombin is involved in the thrombus formation by enzymatic cleavage of two sites of Prothrombin by activated Factor X (Xa) in the clotting process. Single-stranded DNA aptamer that binds to protease thrombin was isolated by researchers that can prohibit fibrin clot formation in-vitro using human plasma [21]. Such aptamers are therapeutically effective for medical conditions such as stroke in which thrombin-catalyzed blood coagulation cascade is involved. Proteinaceous complex resulted from the interaction of certain proteins such as $\alpha$-synuclein, synphilin-1 and parkin in Parkinsons's disease. Ongoing research claims to construct aptamer to target $\alpha$-synuclein aggregation inhibition, which is the cause of Parkinson's disease [22].

Alzheimer's disease is well known neurodegenerative disorder in older age patients. Amyloid- $\beta$ made up of 36-43 amino acids is the main component of amyloid plaques found in the brain of patients with Alzheimer's disease. RNA aptamers constructed against the recombinant prion protein that forms Amyloid- $\beta$ can help stop propagation of Alzheimer's disease [23]. Retroviral Integrase enzyme (HIV-1 Integrase), a potential target in HIV, found in retrovirus enables their DNA to integrate into DNA of the infected cell and thus replicate into host cells. Oligodeoxynucleotide (ODN) aptamers developed by researchers strongly inhibited the integrase enzyme and was proved beneficial therapeutically [24].

Vascular permeability factor (VPF) is a signal protein which promotes vasculogenesis in hypoxic condition and provides blood supply to new or growing tissues. Certain cancer cells express VPF proteins which promote the growth of the tumor and metastasize it. Pegaptanib (marketed as Macugen) is FDA approved RNA aptamer that can inhibit VPF and used as anti-angiogenesis macular degeneration [25]. Subsequently, Pegaptanib was considered for cancer therapy [26]. Keratinocyte growth factor is responsible for facilitating epithelium formation and helps in 
healing. Over-expression of this enzyme results into proliferative disorders of the epithelium. Modified 2'-amino and 2'-fluoro modified pyrimidines containing RNA aptamers were found to inhibit keratinocyte growth factor successfully [27].

Neutrophil elastase is protease enzyme secreted by Neutrophils during inflammation, and it destroys bacteria and host tissues. Over-expression of neutrophil elastase can cause emphysema or emphysematous changes because of the breakdown of the lung structure and increased airspaces. Researchers found that DNA aptamer selected from a pool of random oligonucleotides by SELEX process was efficient to inhibit lung injury in a dosedependent manner [28]. Aptamers are the oligonucleotide chains with higher specificity and affinity towards target protein. Thus, aptamers are considered as a nucleic acid version of antibodies. Various antibodies mediated methods has adopted aptamerbased detection technology. Colorimetric sensors based on aptamer are the revolutionary steps for improving user-friendly tools for end users for homes and clinics. DNA-functionalized gold nanoparticle using DNA aptamers enables colorimetric detection of various compounds such as Adenosine and cocaine. Limitation of this method was precise sample preparation and very low sensitivity [29].

Lateral Flow Assay (LFA) based dry reagent strip biosensor was designed for thrombin detection and quantification using aptamer linked gold nanoparticles. The technology was reported as superior to antibody-based assay and could analyze thrombin with a detection limit of 0.6 pmol. It was also marked that other proteins such as human serum albumin, IgG and IgM were not interfering in analysis demonstrating the specificity of the developed kit [30].

Label-free bioelectronic strategy for measuring target proteins up to femtomolar (fM) concentrations is reported. The invented method is based on ultrasensitive electrochemical measurement of guanine-rich secondary aptamer with or without amplification using polymerase chain reaction. Quantification of purine bases is extremely useful monitoring of DNA hybridization [31]. Realtime analysis of protein at the amount as low as single molecule was developed by the researchers using single-walled carbon nanotubes (SWNTs). Individual DNA aptamers were coupled with SWNT at point contact to form the single-molecule device. This device allows reversible and selective detection of a single molecule of analyte thrombin [32].

Aptamers can be used for targeted drug delivery for site specific therapeutic action. Nucleolin is an abundant phosphoprotein present in Nucleolus. Recently, nucleolin is found on the surface of rapidly proliferating cells. Because of its extracellular location, nucleolin is preferred target for anti-cancer drugs. The drug can be conjugated with Nucleolin specific aptamer which can selectively bind with Nucleolin. Upon administration of this conjugate into the body, aptamer will bind specifically to Nucleolin located on rapidly proliferating cells and the drug can be released at the site of action. Recent research claims Nucleolin-aptamer therapy to cure retinoblastoma [33]. In another study, Nucleolin specific aptamers were conjugated with cationic liposomes containing anti-BRAF siRNA (BRAF is the mutated gene in $60 \%$ of malignant melanomas). Small interfering RNA selectively bind to BRAF to inhibit its expression [34].

Quantification of single molecule protein present on the outer cell membrane is reported using Fluorescence correlation spectroscopy (FCS), specifically for receptor proteins. In that study, Aptamers were designed to bind to outer membrane proteins of $S$. typhimurium and were measured for specificity against Escherichia coli. Aptamer probes were fluorescently labeled and exposed to FCS so that they could study the diffusion dynamics of bound and unbound aptamers and could link them to determine the dissociation constants and receptor densities of the bacteria for each aptamer at very low concentration (at single molecule level) [35].

Aptamers have properties of high specificity and affinity for a particular protein that can be used for protein purification (downstream processing). Histidine (His)-tagged proteins were separated using affinity chromatography by immobilizing Histagged aptamers on magnetic beads linked by covalent bonds. His-tagged proteins were separated from complex lysate of $E$. Coli. These aptamer-based magnetic beads can be regenerated and reused for purification [36].

Novel use of Aptamers has been reported to silent the genes responsible for cancer. The sequence of the gene responsible for expression and subsequent proliferation of cells were identified. Complementary sequence of such mRNA sequence was constructed as aptamer oligonucleotide, which may be administered into the body by novel drug delivery system to target cell nucleus. The designed Aptamer will bind to the complementary sequence of mRNA and block the translation of such gene acting as 'Antisense Aptamers' [37,38].

RNA-aptamer conjugated liposomes were designed to target the prostate-specific membrane antigen (PSMA) expressed on the cell membrane of prostate cancer cells. Researchers referred this liposome as 'Aptamosomes or Aptasome'. Doxorubicin encapsulated liposomes were conjugated with aptamers on the outer membrane that are more toxic specifically to cancer cells. Toxicity of Doxorubicin can be reduced by targeting it at the site of action and reducing systemic exposure [39]. Aptamers have been utilized for environment monitoring and early warning applications also. Incredibly increasing pollutants encouraged the immediate need of innovative, safe, accurate, rapid and user-friendly technology for environment monitoring. Optical biosensors are developed using functional biorecognition materials such as Aptamers to detect, identify and quantify the biological and other contaminants (e.g. virus, enzymes, heavy metals, toxins, persistent organic pollutant, endocrine disrupting chemicals, etc.) [40].

\section{Conclusion}

The present review discusses the overview of aptamers starting from manufacturing to therapeutic and diagnostic applications. Studies done for Aptamer research have been proved to be practically feasible and effective. Aptamers are the bridge between simple chemical molecules and complex protein drug products. The affinity of aptamers for target protein makes them competitive with the antibody. However, chemical 
synthesis, physio-chemical stability, high affinity and specificity makes Aptamer a potential candidate to distinguish future of biotechnology.

\section{Conflicts of Interest}

The authors declare no conflict of interest.

\section{Acknowledgment}

There were no funding sources for the study.

\section{References}

1. Woodcock J, Griffin J, Behrman R, Cherney B, Crescenzi T, et al. (2007) The FDA's assessment of follow-on protein products: a historical perspective. Nat Rev Drug Discov 6(6): 437-442.

2. Nemoto N, Tsutsui C, Yamaguchi J, Ueno S, Machida M, et al. (2012) Antagonistic effect of disulfide-rich peptide aptamers selected by cDNA display on interleukin-6-dependent cell proliferation. Biochem Biophys Res Commun 421(1): 129-133.

3. Brimacombe R, Trupin J, Nirenberg M, Leder P, Bernfield M, et al. (1965) RNA Codewords and Protein Synthesis: The Nucleotide Sequences of Multiple Codewords for Phenylalanine, Serine, Leucine, and Proline. Science 147(3657): 479-484.

4. Wang W (2015) Advanced protein formulations. Protein Sci 24(7): 1031-1039.

5. Radi A (2011) Electrochemical Aptamer-Based Biosensors: Recent Advances and Perspectives. International Journal of Electrochemistry 2011(2011): 1-17.

6. Ruscito A, DeRosa M (2016) Small-Molecule Binding Aptamers: Selection Strategies, Characterization, and Applications. Front Chem $4: 1-14$.

7. Schütze T1, Wilhelm B, Greiner N, Braun H, Peter F, et al. (2011) Probing the SELEX Process with Next-Generation Sequencing. PLoS One 6(12): e29604.

8. Famulok M, Szostak J (1992) In Vitro Selection of Specific Ligandbinding Nucleic Acids. Angewandte Chemie International Edition in English 31(8): 979-988.

9. Wright W, Funk W (1993) CASTing for multicomponent DNA-binding complexes. Trends Biochem Sci 18(3): 77-80.

10. Tolle F, Wilke J, Wengel J, Mayer G (2014) By-Product Formation in Repetitive PCR Amplification of DNA Libraries during SELEX. PLoS One 9(12): e114693.

11. Zhong J (2013) Future Trends in Biotechnology. ( $1^{\text {st }}$ edn), Springer, USA, pp. 153-169.

12. Christopher P Rusconi, Elizabeth Scardino, Juliana Layzer, George A Pitoc, Thomas L Ortel, et al. (2002) RNA aptamers as reversible antagonists of coagulation factor IXa. Nature 419(6902): 90-94.

13. Burmeister PE, Lewis SD, Silva RF, Preiss JR, Horwitz LR, et al. (2005) Direct In Vitro Selection of a 2'-0-Methyl Aptamer to VEGF. Chem Biol 12(1): 25-33.

14. Keefe A, Pai S, Ellington A (2010) Aptamers as therapeutics. Nat Rev Drug Discov 9(7): 537-550.
15. Hu PP, Zhang K (2015) The modulation of coagulation by aptamers. Blood Coagu Fibrinolysis 26(1): 1-6.

16. Sooter L, Ellington A (2002) Reflections on a Novel Therapeutic Candidate. Chemistry Biology 9(8): 857-858.

17. Walsh R, DeRosa M (2009) Retention of function in the DNA homolog of the RNA dopamine aptamer. Biochem Biophys Res Commun 388(4): 732-735.

18. Chang TW, Blank M, Janardhanan P, Singh BR, Mello C, et al. (2010) In vitro selection of RNA aptamers that inhibit the activity of type A botulinum neurotoxin. Biochem Biophys Res Commun 396(4): 854860.

19. Bruno JG, Richarte AM, Carrillo MP, Edge A (2012) An aptamer beacon responsive to botulinum toxins. Biosens Bioelectron 31(1): 240-243.

20. McConnell E, Holahan M, DeRosa M (2014) Aptamers as Promising Molecular Recognition Elements for Diagnostics and Therapeutics in the Central Nervous System. Nucleic Acid Ther 24(6): 388-404.

21. Bock LC, Griffin LC, Latham JA, Vermaas EH, Toole JJ (1992) Selection of single-stranded DNA molecules that bind and inhibit humanthrombin. Nature 355(6360): 564-566.

22. Utilization of Aptamers to Prevent Protein Aggregation in Parkinson's disease. The Michael J Fox Foundation for Parkinson's Research.

23. Rahimi F, Bitan G (2010) Selection of Aptamers for Amyloid \&beta; Protein, the Causative Agent of Alzheimer's Disease. J Vis Exp (39): 10.3791 .

24. de Soultrait VR, Lozach PY, Altmeyer R, Tarrago-Litvak L, Litvak S, et al. (2002) DNA Aptamers Derived from HIV-1 RNase H Inhibitors are Strong Anti-integrase Agents. J Mol Biol 324(2): 195-203.

25. NG E, ADAMIS A (2006) Anti-VEGF Aptamer (Pegaptanib) Therapy for Ocular Vascular Diseases. Annals of the New York Academy of Sciences 1082(1): 151-171.

26. Zhou G, Wilson G, Hebbard L, Duan W, Liddle C, et al. (2016) Aptamers: A promising chemical antibody for cancer therapy. Oncotarget 7(12): 13446-13463.

27. Pagratis NC, Bell C, Chang YF, Jennings S, Fitzwater T, et al. (1997) Potent 2'-amino-, and 2'-fluoro-2'-deoxyribonucleotide RNA inhibitors of keratinocyte growth factor. Nat Biotechnol 15(1): 68-73.

28. Bless NM, Smith D, Charlton J, Czermak BJ, Schmal H, et al. (1997) Protective effects of an aptamer inhibitor of neutrophil elastase in lung inflammatory injury. Curr Biol 7(11): 877-880.

29. Radi A (2011) Electrochemical Aptamer-Based Biosensors: Recent Advances and Perspectives. International Journal of Electrochemistry 2011(1): 1-17.

30. Mao X, Xu H, Zeng Q, Zeng L, Liu G (2009) Molecular beaconfunctionalized gold nanoparticles as probes in dry-reagent strip biosensor for DNA analysis. Chem Commun (Camb) (21): 3065-3067.

31. Xiang Y, Xie M, Bash R, Chen J, Wang J (2007) Ultrasensitive LabelFree Aptamer-Based Electronic Detection. Angew Chem Int Ed Engl 46(47): 9212-9214.

32. Liu S, Zhang X, Luo W, Wang Z, Guo X, et al. (2011) Single-Molecule Detection of Proteins Using Aptamer-Functionalized Molecular Electronic Devices. Angew Chem Int Ed Engl 50(11): 2544-2550. 
33. Subramanian N, Srimany A, Kanwar JR, Kanwar RK, Akilandeswari B, et al. (2016) Nucleolin-aptamer therapy in retinoblastoma: molecular changes and mass spectrometry-based imaging. Mol Ther Nucleic Acids 5(8): e358.

34. Li L, Hou J, Liu X, Guo Y, Wu Y, et al. (2014) Nucleolin-targeting liposomes guided by aptamer AS1411 for the delivery of siRNA for the treatment of malignant melanomas. Biomaterials 35(12): 38403850 .

35. Book B, Chen J, Irudayaraj J (2011) Quantification of receptor targeting aptamer binding characteristics using single-molecule spectroscopy. Biotechnol Bioeng 108(5): 1222-1227.

36. Kökpinar Ö, Walter JG, Shoham Y, Stahl F, Scheper T (2011) Aptamerbased downstream processing of his-tagged proteins utilizing magnetic beads. Biotechnol Bioeng 108(10): 2371-2379.
37. Burnett J, Rossi J (2012) RNA-Based Therapeutics: Current Progress and Future Prospects. Chem Biol 19(1): 60-71.

38. Fattal E, Bochot A (2006) Ocular delivery of nucleic acids: antisense oligonucleotides, aptamers and siRNA. Adv Drug Deliv Rev 58(11): 1203-1223.

39. Baek SE, Lee KH, Park YS, Oh DK, Oh S, et al. (2014) RNA aptamerconjugated liposome as an efficient anticancer drug delivery vehicle targeting cancer cells in vivo. J Control Release 196: 234-242.

40. Long F, Zhu A, Shi H (2013) Recent Advances in Optical Biosensors for Environmental Monitoring and Early Warning. Sensors (Basel) 13(10): 13928-13948. 